International Journal of Engineering \& Technology, $7(2.20)(2018)$ 105-108
International Journal of Engineering \& Technology
SPC
Website: www.sciencepubco.com/index.php/IJET
Research paper

\title{
A Secured IoT Based Advanced Health Care System for Medical Field using Sensor Network
}

\author{
Paparao Nalajala ${ }^{1 *}$, S Bhagya Lakshmi ${ }^{2}$ \\ ${ }^{1}$ Department of Electronics and Communication Engineering, Institute of Aeronautical Engineering, \\ Hyderabad, India-500043 \\ ${ }^{2}$ Department of Electronics and Communication Engineering, M.Tech Student, Institute of Aeronautical Engineering, \\ Hyderabad, India-500043 \\ *Corresponding author E-mail: nprece@gmail.com
}

\begin{abstract}
In day to day life health awareness and controlling system is important to monitor those patient's physiological parameters frequently. In the recent health awareness environment those usage of IOT innovation organization acquires accommodation of professionals and also patients, since they are connected to different medical fields. A sensor node needs to be arranged on the apparent of the patient body will gather the entire signal from those wireless sensor and also send them of the body sensor node. Those connected sensor nodes on the patient's body can make in the structure of a wireless body sensor network furthermore they have the capacity with sense those heart beat rate, temperature of surroundings. That basic focus to this system may be on transmitting the individuals' patient's health watching parameters through wireless communication in crucial conditions. We propose a secure IOT built health awareness monitoring and will check that saline level of the patient.
\end{abstract}

Keywords: Internet of things (IOT), Sensors, Saline bottle, data confidentiality, data reliability and authentication.

\section{Introduction}

The sensor network innovation is one of the most crucial technologies used in the IOT based health care monitoring system [1]. The human body capacities and encompassing environment is monitor by using the low-powdered and light weight wireless sensor hubs. The BSN nodes will efficiently look delicate (life critical) majority of the data. According to the recent survey many aged people will suffers as of at most one long-lasting disease and the old persons cannot take care of them. The body sensor node helps to provide information about the healthcare services like medical data access and medical monitoring and communication with the family members and with the doctor in emergency situation through the SMS [1].The body sensor networks enhance the quality of the life.

Internet of thing is the most important communication in the $21 \mathrm{st}$ century. In the environment of IoT all objects of internet communication and computing capabilities such as micro controller, transceivers for digital communication play a major role in our day to day. IOT includes a significant number sort of sensors that empower a significant number of aged people to enjoy the modern health care services anywhere, anytime. The smart device uses are used to gather temperature, blood pressure, heart beat and saline bottle level which are used to evaluate the health condition of the patient [1-3].

In this project we have the temperature, blood pressure, ECG and heart beat and saline bottle level would monitored eventually by using ARM7 and is measured utilizing particular sensors and it can be in the screen of the computer and will be monitoring anywhere in the world using internet source. BSN care node will col- lect and store the data in the processing in local processing unit and it sends to database server. The internet link and power source must be in the constant requisite in accurate working and once in a while observing physiology parameter of body to avoid hazard. Since the sensor is detecting or gathering the data once each 10 seconds and drives to database server.

\section{Literature Review}

Sensor network is the key advances for those improvements of IOT in the health care system, over which a sick man could make analyzed utilizing an accumulation of light hubs and sensor light. However the advancement for this new framework done in health care requisitions without regards to safety makes the security for patients vulnerable. In this article they firstly focuses on the safety requirement in the modern health care BSN[1].The device will detects if a patient is in disorder to the doctor and Rx connected to a computer diagram chart for monitored physiological specification of the human bodyb[4].

Those sensors utilized are temperature sensor, pulse sensor, heart beat sensor. These sensors would be arranged on the human body which need aid help to monitor the health state without irritating that daily calendar of the patient. The data will be sent of the physician server for the long extent wireless system utilizing GSM. The health checking system holds those microcontroller, sensors, GSM modem LCD presentation on transmit or receive health related information of the specialist. Similarly the same GSM modem is used in the hospital. 


\section{Existing System}

The improvement in the body sensor network in the health care application had prepared patient observing more easily. Recently several researcher and project have been proposed in which have been proposed in which it provides continuous patient monitoring in open environment.

This Existing system will provide a few popular researches in healthcare system. Code blue [6] [7] is a health care scientific project based on BSN and was developed at Harvard sensor network lab. In this existing project several sensors are arranged on the surface of the patient's body which could sense the body and transmit the data wireless to the end user. However, code blue's author acknowledges the need of security in medical applications, but security is still a major problem in this project. Alarm-net comprises of body sensor networks and environmentally friendly sensor networks.

The goal of this object was to report the concerns notable for convention regarding wearable and implantable sensors for dispersed mobile monitoring. In spite of NG et al. Suggested and showed the universal health care monitoring architecture, it may be for the most part recognized that without distinguishing the security to wireless health care monitoring, which might make an essential prerequisite for human services provisions Concerning illustration expressed Toward legislature laws. [8].

\section{Proposed System}

In this paper the proposed method uses the Lpc2148 controller as a gateway to communicate with the various sensors like temperature sensor, heart beat sensor and saline bottle level and sends the evidence to the net page and then plot the sensor data in graphical statistics. The information will be updated from the implemented system and the doctor can be accessible anywhere from the world through the internet. If any sensor get the abnormal condition the message alert will be sends to the predefine mobile number.

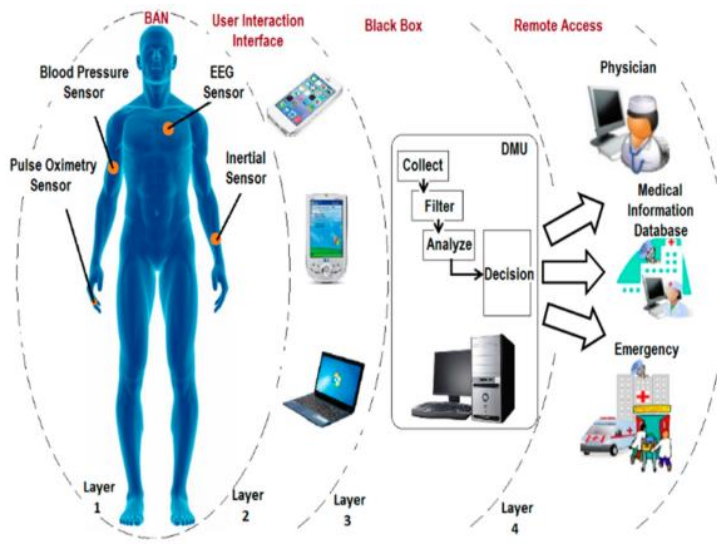

Figure.1. System Design Architecture

The security issue can be addressed by setting a password the user can only open the web page and can know the information in the critical situation through GSM the data or the SMS alert will be send to the predefined mobile number. The proposed system deals with the patient health monitoring conditions such as temperature, heartbeat, MEMS accelerometer and saline level with sensors and informs the data to the web server for plotting the sensor information in graphical statistics. We can access the data which is updated from the implemented system and can be accessible through the internet from anywhere in the world and if any sensors gets abnormal condition then we will get SMS alert to predefine mobile number [7]

\subsection{Heart Beat Sensor}

Those heart beat sensor is used to measure those heartbeat of the tolerant it provides for those advanced digital output of heart beat that points when finger may be set on it. It could have a chance to be decreased in size. Those attempting voltage of heart beat will be $+5 \mathrm{v}$ DC. It meets expectations on the statement, by the blood flow through finger at every pulse by utilizing light regulation. The range of a heartbeat sensor varies between $60-100$ beats per minute. When the pulse range exceeds 100 LED flash will get into on condition by giving digital output directly to microcontroller for measuring the beats per minute (BPM).

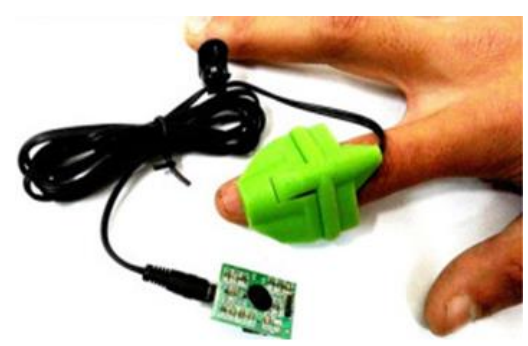

Figure 2.1 Hearts Beat Sensor

\subsection{Temperature Sensor}

Temperature sensor is incorporated information preparing which will be used to measure temperature with a produce electrical energy linearly proportionate of the centigrade temperature. The LM35 sensor essential positive element through straight temperature sensor, concerning illustration those client need not to aggravate those transformation from claiming kelvin with centigrade. Those LM35 will be straightforwardly over Celsius What's more it will be likewise utilized in the remote requisitions. Temperature may be a standout amongst the greater part by and large measured parameter in the globe [9-11].

There are two types of temperature sensors.

Non-contact Temperature sensors: Non conduct temperature sensor utilizes radiation and convection to monitor temperature.

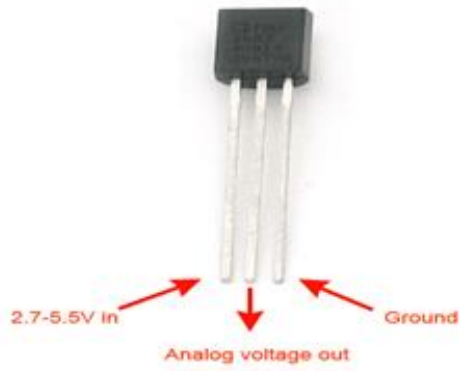

Figure.3. Temperature Sensor

\subsection{MEMS Accelerometer}

Micro-Electro-Mechanical System (MEMS) descried all categories of units and methods for manufacturing .This tiny material have the ranging from 1-100 microns. MEMS devices are generally categorized into two types' sensors and actuators. Sensors devices gather information from their surroundings and actuators will execute the commands. Accelerometer measures acceleration by changing capacitance. The mass is fixed and capacitor is variable.

MEM is a complete system-on-chip technology. Electrical process the data and Mechanical acts on data. MEM is a complete integrated circuit thinking part of a system. MEM is a perception and control function .MEMS technology extensively used in many applications.

MEMS is one type of sensor which can senses the person's effort and if there is an irregular movement such as sinking down on the surface can be detected. The measured information can be sent 
through the SMS alert. MEMS act as a key component which can sense or detect person movement and variation in blood pressure. The MEMS is the slight dimension and ultra-low power consumption because of this application it can also lead to improvement in the telemedicine

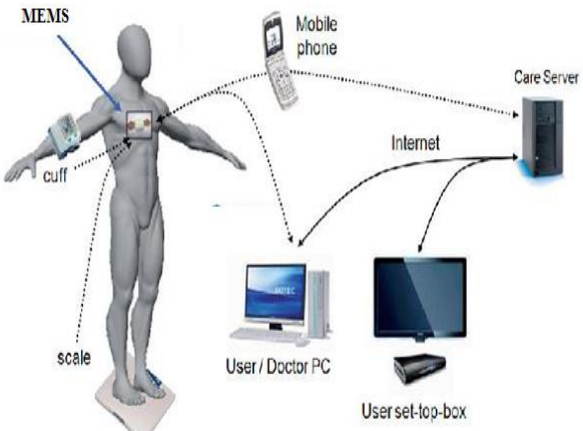

Figure.4. MEMS working Operations in System

\subsection{Saline Bottle Level Monitoring}

In our project the infrared sensor will measure saline level. The normal saline bottle is of $1000 \mathrm{ml}$.If the saline level is in critical point then the SMS alert will be send to the predefined mobile number. The nurse can come and change saline bottle.

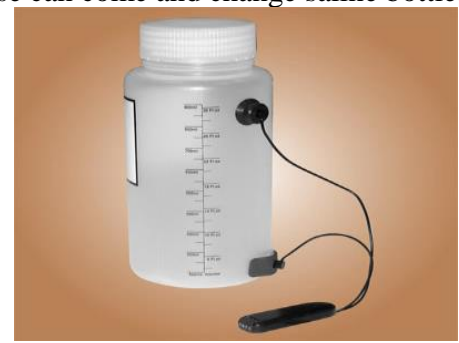

Figure.4. Saline Level Monitoring

\subsection{Internet of Things}

The internet of things is a developing network of physical objects which has a physical connectivity and communication through IP address between objects and internet enable devices [1]

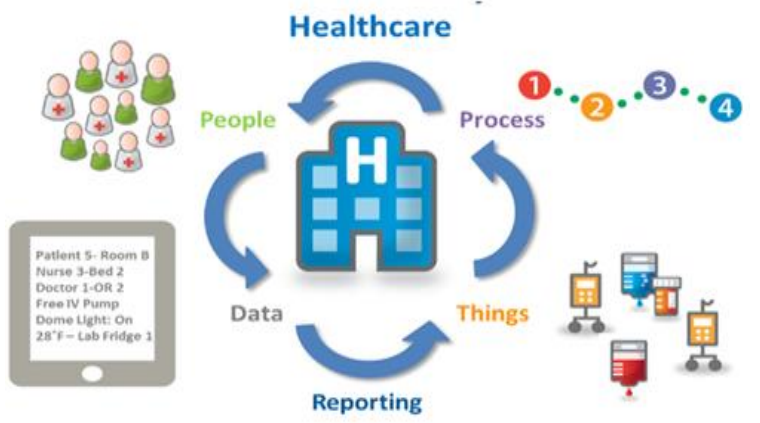

Figure.5. Internet of Things

The proposed system contains the ESP8266 is the Wi-Fi module which is used to connected to the server. So that the Wi-Fi network through IOT the data will be reads on the webpage. In order to make a simple TCP/IP connection using Hayes-style commands $\mathrm{Wi}$-Fi network is connected to a small module i.e microcontroller. The ESP8266 is an ESP8266 with 1 MB about inherent flash, permitting g to single-chip devices capable of interfacing to Wi-Fi [7][12]

\section{Design Flow Chart}

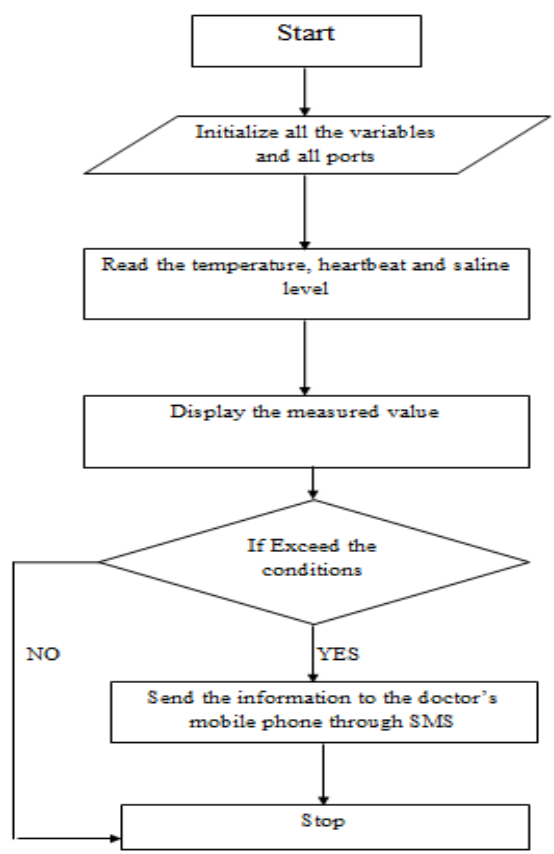

Figure.6. System Design Flow Chart

\section{Result Analysis}

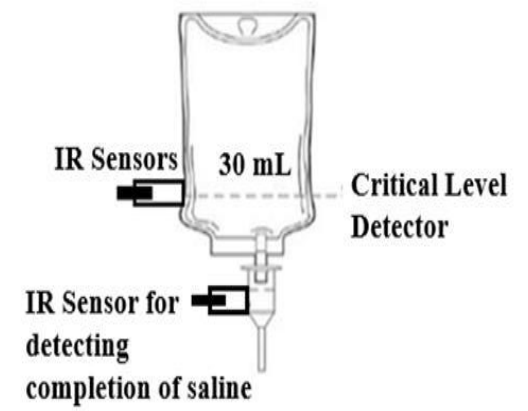

Figure.7 . Saline bottle level

When the saline level reaches critical level then SMS alert will be send to the predefined mobile.

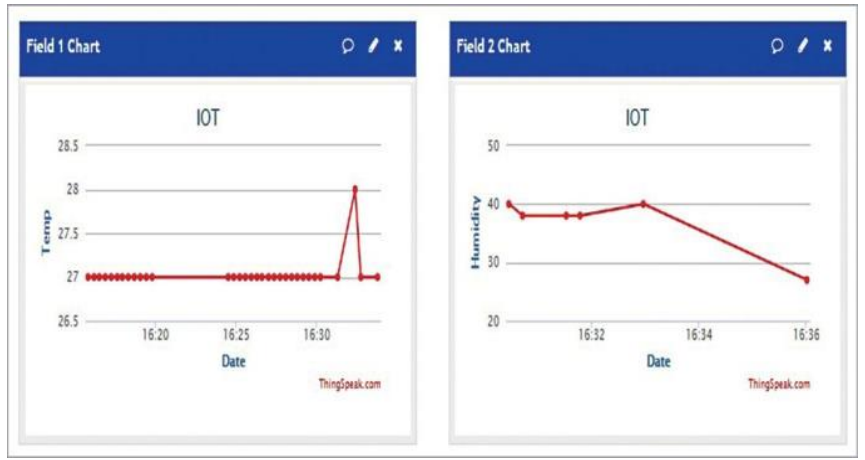

Figure.7.1.Temperature Sensor Graphical Representation 


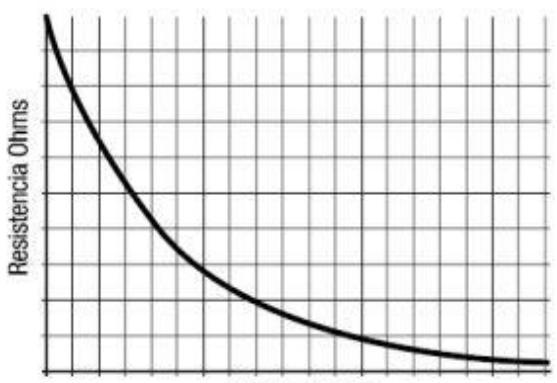

Temperatura ${ }^{\circ} \mathrm{C}$

Figure.7.2. The graph shows the temperature is linearly proportional to the Celsius

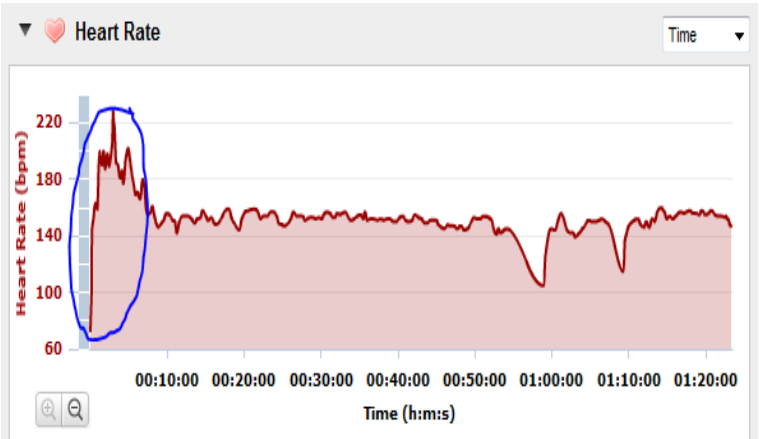

Figure.7.3. Heart Beat Sensor Graphical Representation

When the heart beat is in critical condition i.e. the circled parts the SMS alert will be send to the predefined mobile

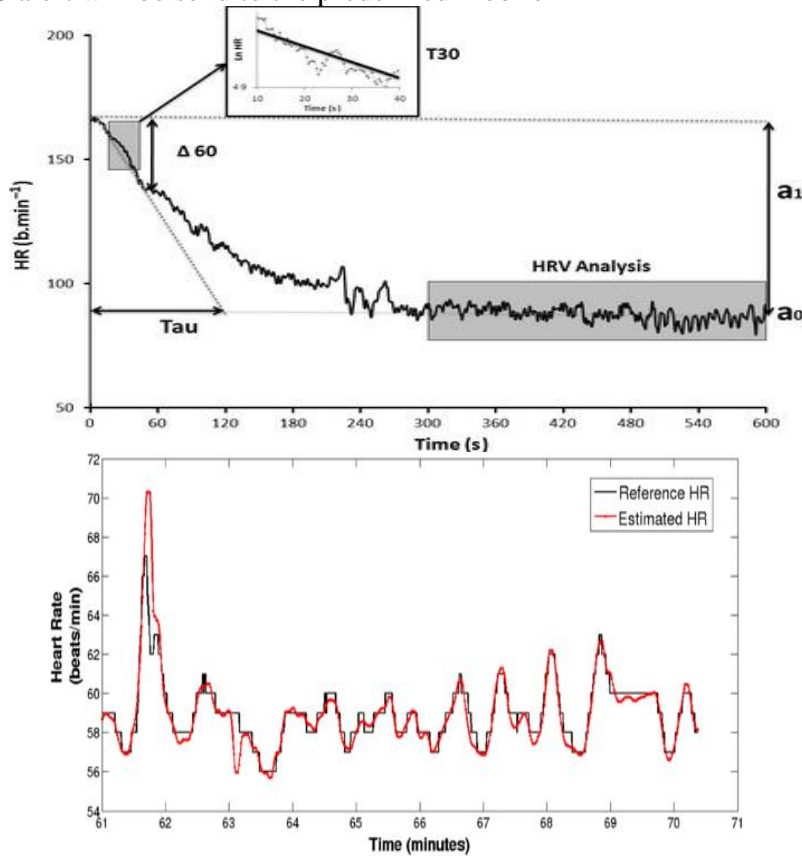

Figure.7.4. The variation of the heartbeat of a person.

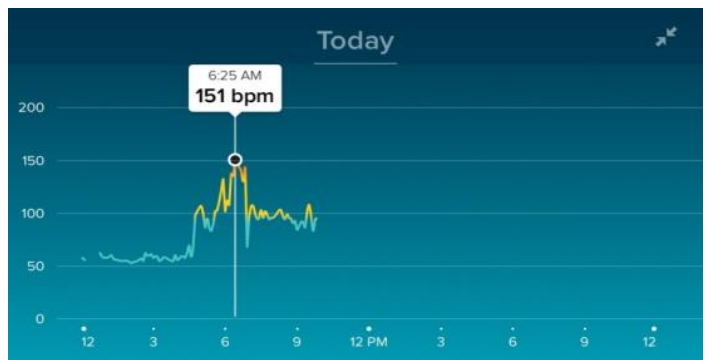

Figure.7.5. The heart beat rate along with time will be send to the web page.

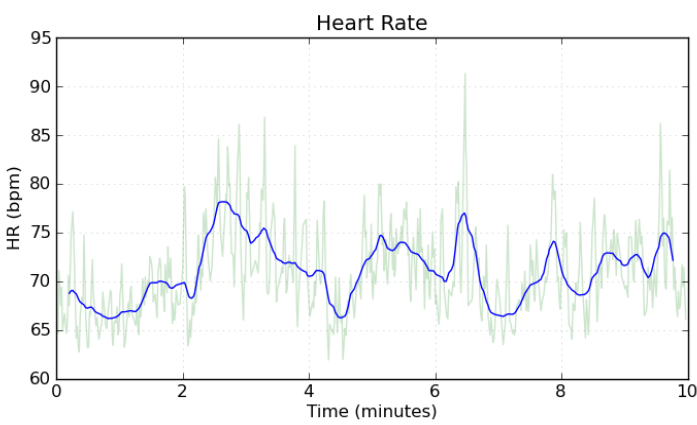

\section{Conclusion}

In this object at mainly we must depicted the security and the protection issues in human services requisition utilizing sensor network. Successively we establish that in spite of the information that the vast majority of the popular different medical sensors based exploration projects recognize the issues of the safety, and then they failed to drive in strong safety services that might be preserved patient confidentiality.

\section{References}

[1] Prosanta Gope and Tzonelih Hwang," BSN-Care: A Secure IoTBased Modern Healthcare System Using Body Sensor Network", IEEE SENSORS JOURNAL, VOL. 16, NO. 5, MARCH 1, 2016.

[2] P. Gope and T. Hwang, "Untraceable sensor movement in distributed IoT infrastructure," IEEE Sensors J., vol. 15, no. 9, pp. 5340-5348, Sep. 2015

[3] P. Kumar and H.-J. Lee, "Security issues in healthcare applications using wireless medical sensor networks: A survey," Sensors, vol. 12, no. 1,pp. 55-91, 2012.

[4] Bhavana Godavarthi, Mohammad Khadir, Y Mrudula, K Nishanth Rao, Paparao Nalajala," Biomedical Sensors Based Remote Monitoring System Field Of Medical And Health Care", Journal of Advanced Research in Dynamical and Control Systems Vol. 9, Issue 4 ,OCT.2017

[5] K. Lorincz et al., "Sensor networks for emergency response: Challenges and opportunities," IEEE Pervasive Comput., vol. 3, no. 4, pp. 16-23 Oct./Dec. 2004.

[6] A. Wood et al., "ALARM-NET: Wireless sensor networks for assisted living and residential monitoring," Dept. Comput. Sci., Univ. Virginia, Charlottesville, VA, USA, Tech. Rep. CS-200601, 2006.

[7] Bhavana Godavarthi, Paparao Nalajala," Design and Implementation of Vehicle Navigation System in Urban Environments using Internet of Things (IoT)", IOP Conf. Series: Materials Science and Engineering 225 (2017) 012262.

[8] S. Pai et al., "Confidentiality in sensor networks: Transactional information," IEEE Security Privacy Mag., vol. 6, no. 4, pp. 28 35, Jul./Aug. 2008.

[9] J. W. P. Ng et al., "Ubiquitous monitoring environment for wearable and implantable sensors (UbiMon)," in Proc. 6th Int. Conf. Ubiquitous Comput. (UbiComp), Nottingham, U.K., Sep. 2004, pp. 1-2.

[10] Office for Civil Rights. United State Department of Health and Human Services Medical Privacy. National Standards of Protect the Privacy of Personal-Health-Information

[11] J. Ko et al., "MEDiSN: Medical emergency detection in sensor networks," ACM Trans. Embed. Comput. Syst., vol. 10, no. 1, pp. 1-29, Aug. 2010.

[12] Paparao Nalajala, Bhavana Godavarthi," Working Women Hand Held Safety Self Defense System using IoT", Journal of Advanced Research in Dynamical and Control Systems, 18Special Issue, Year: 2017, Pages::2051-2059.

[13] Paparao Nalajala, Bhavana Godavarth, M Lakshmi Raviteja, Deepthi Simhadri," Morse code Generator Using Microcontroller with Alphanumeric Keypad", International Conference on Electrical, Electronics, and Optimization Techniques (ICEEOT), Pages 762 - 766,Publisher-IEEE 